\title{
Tarski Geometry Axioms. Part IV - Right Angle
}

\author{
Roland Coghetto \\ Rue de la Brasserie 5 \\ 7100 La Louvière, Belgium
}

\author{
Adam Grabowski \\ Institute of Informatics \\ University of Białystok \\ Poland
}

\begin{abstract}
Summary. In the article, we continue [7] the formalization of the work devoted to Tarski's geometry - the book "Metamathematische Methoden in der Geometrie" (SST for short) by W. Schwabhäuser, W. Szmielew, and A. Tarski 14, 9, 10. We use the Mizar system to systematically formalize Chapter 8 of the SST book.

We define the notion of right angle and prove some of its basic properties, a theory of intersecting lines (including orthogonality). Using the notion of perpendicular foot, we prove the existence of the midpoint (Satz 8.22), which will be used in the form of the Mizar functor (as the uniqueness can be easily shown) in Chapter 10. In the last section we give some lemmas proven by means of Otter during Tarski Formalization Project by M. Beeson (the so-called Section 8A of SST).
\end{abstract}

MSC: 51A05 51M04 68T99 03B35

Keywords: Tarski geometry; foundations of geometry; right angle

MML identifier: GTARSKI4, version: 8.1.09 5.54.1344

\section{INTRODUCTION}

We use the Mizar system [1, 2] to systematically formalize Chapter 8 ("Rechte Winkel - Right angle") of the SST book. The theorems of this chapter are valid in neutral geometry [13].

We start (Def. 1) with the translation of the definition of the "right angle" which in SST reads as follows: 
$a, b, c$ bilden einen rechten winkel (mit dem Scheitel $b$ ):

$$
R a b c: \longleftrightarrow a c \equiv a S_{b}(c) .
$$

In the Mizar formalism (note explicit use of Tarski's axioms):

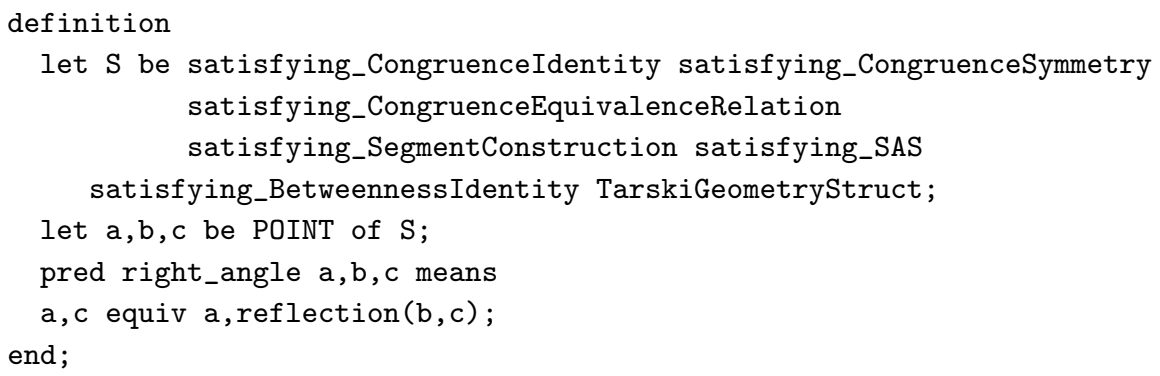

where reflection is defined in [7].

For the purpose of this presentation, we use the notation $b(a, b, c)$ instead of Rabc chosen in SST. Section 3 starts with variants of Definition 8.11, while in the next section predicate $A, B$ Is $x$ is defined, and this is Def. 7 in our translation. Section 5 deals with perpendicular foot - Satz 8.18 is Lotsatz, Satz 8.22 states that every segment has a midpoint (Gupta 1965 [11]).

In 2006, the first eight chapters were formalised in Coq in 2006 by Narboux 12 and we are essentially in this place. The entire SST book have been formalized within intuitionistic logic [5]. Note that the definitions in [6]

(* Definition 8.1.*)

Definition Per A B C := exists C', Midpoint B C C' $\bigwedge$ Cong A C A C'.

and in [4]: $A B C$ is a right angle if there is a point $D$ such that $\mathbf{B}(A, B, D)$ and $A B=D B$ and $A C=D C$ :

rightangle 'RR A B C $\Leftrightarrow$ ?X. BE A B X $八 \mathrm{EE} \mathrm{A} \mathrm{B} \mathrm{X} \mathrm{B} \mathrm{八} \mathrm{EE} \mathrm{A} \mathrm{C} \mathrm{X} \mathrm{C} \mathrm{ハ} \mathrm{NE} \mathrm{B} \mathrm{C'}$

are slightly different than in SST.

Some of the results were obtained by means of other automatic proof assistants, either partially [8], or completely [3].

\footnotetext{
${ }_{1}^{1}$ https://github.com/GeoCoq/GeoCoq/blob/master/Tarski_dev/Definitions.v
} 


\section{Preliminaries}

From now on $S$ denotes a non empty Tarski plane satisfying seven Tarski's geometry axioms and $a, b, c, d, c^{\prime}, x, y, z, p, q, q^{\prime}$ denote points of $S$.

Let $S$ be a non empty Tarski plane satisfying the axiom of congruence identity, the axiom of segment construction, the axiom of betweenness identity, and the axiom of Pasch and $a, b$ be points of $S$. Let us note that the functor $\operatorname{Line}(a, b)$ is commutative.

Now we state the proposition:

(1) Let us consider Tarski plane $S$ satisfying the axiom of congruence symmetry, the axiom of congruence equivalence relation, and the axiom of congruence identity, and points $a, b, c, d$ of $S$. Suppose $\overline{a b} \cong \overline{c d}$. Then

(i) $\overline{a b} \cong \overline{d c}$, and

(ii) $\overline{b a} \cong \overline{c d}$, and

(iii) $\overline{b a} \cong \overline{d c}$, and

(iv) $\overline{c d} \cong \overline{a b}$, and

(v) $\overline{d c} \cong \overline{a b}$, and

(vi) $\overline{c d} \cong \overline{b a}$, and

(vii) $\overline{d c} \cong \overline{b a}$.

Let us consider Tarski plane $S$ satisfying the axiom of congruence symmetry, the axiom of congruence equivalence relation, and the axiom of congruence identity and points $p, q, a, b, c, d$ of $S$. Now we state the propositions:

(2) Suppose $(\overline{p q} \cong \overline{a b}$ or $\overline{p q} \cong \overline{b a}$ or $\overline{q p} \cong \overline{a b}$ or $\overline{q p} \cong \overline{b a})$ and $(\overline{p q} \cong \overline{c d}$ or $\overline{p q} \cong \overline{d c}$ or $\overline{q p} \cong \overline{c d}$ or $\overline{q p} \cong \overline{d c})$. Then

(i) $\overline{a b} \cong \overline{d c}$, and

(ii) $\overline{b a} \cong \overline{c d}$, and

(iii) $\overline{b a} \cong \overline{d c}$, and

(iv) $\overline{c d} \cong \overline{a b}$, and

(v) $\overline{d c} \cong \overline{a b}$, and

(vi) $\overline{c d} \cong \overline{b a}$, and

(vii) $\overline{d c} \cong \overline{b a}$.

The theorem is a consequence of (1).

(3) Suppose $(\overline{p q} \cong \overline{a b}$ or $\overline{p q} \cong \overline{b a}$ or $\overline{q p} \cong \overline{a b}$ or $\overline{q p} \cong \overline{b a}$ or $\overline{a b} \cong \overline{p q}$ or $\overline{b a} \cong \overline{p q}$ or $\overline{a b} \cong \overline{q p}$ or $\overline{b a} \cong \overline{q p})$ and $(\overline{p q} \cong \overline{c d}$ or $\overline{p q} \cong \overline{d c}$ or $\overline{q p} \cong \overline{c d}$ or $\overline{q p} \cong \overline{d c}$ or $\overline{c d} \cong \overline{p q}$ or $\overline{d c} \cong \overline{p q}$ or $\overline{c d} \cong \overline{q p}$ or $\overline{d c} \cong \overline{q p})$. Then 
(i) $\overline{a b} \cong \overline{d c}$, and

(ii) $\overline{b a} \cong \overline{c d}$, and

(iii) $\overline{b a} \cong \overline{d c}$, and

(iv) $\overline{c d} \cong \overline{a b}$, and

(v) $\overline{d c} \cong \overline{a b}$, and

(vi) $\overline{c d} \cong \overline{b a}$, and

(vii) $\overline{d c} \cong \overline{b a}$, and

(viii) $\overline{a b} \cong \overline{c d}$.

The theorem is a consequence of (1) and (2).

(4) Let us consider Tarski plane $S$ satisfying the axiom of congruence identity, the axiom of segment construction, the axiom of betweenness identity, and the axiom of Pasch, and points $a, b$ of $S$. Then

(i) $a, b$ and $b$ are collinear, and

(ii) $b, b$ and $a$ are collinear, and

(iii) $b, a$ and $b$ are collinear.

(5) Let us consider a non empty Tarski plane $S$ satisfying seven Tarski's geometry axioms, and points $p, q, r$ of $S$. Suppose $p \neq q$ and $p \neq r$ and $(p$, $q$ and $r$ are collinear or $q, r$ and $p$ are collinear or $r, p$ and $q$ are collinear or $p, r$ and $q$ are collinear or $q, p$ and $r$ are collinear or $r, q$ and $p$ are collinear). Then

(i) $\operatorname{Line}(p, q)=\operatorname{Line}(p, r)$, and

(ii) Line $(p, q)=\operatorname{Line}(r, p)$, and

(iii) Line $(q, p)=\operatorname{Line}(p, r)$, and

(iv) $\operatorname{Line}(q, p)=\operatorname{Line}(r, p)$.

(6) Let us consider a Tarski plane $S$, and points $a, b, c$ of $S$. Suppose Middle $(a, b, c)$ or $b$ lies between $a$ and $c$. Then $a, b$ and $c$ are collinear.

(7) Let us consider Tarski plane $S$ satisfying the axiom of congruence identity, the axiom of segment construction, the axiom of betweenness identity, and the axiom of Pasch, and points $a, b, c$ of $S$. Suppose Middle $(a, b, c)$ or $b$ lies between $a$ and $c$. Then

(i) $a, b$ and $c$ are collinear, and

(ii) $b, c$ and $a$ are collinear, and

(iii) $c, a$ and $b$ are collinear, and

(iv) $c, b$ and $a$ are collinear, and 
(v) $b, a$ and $c$ are collinear, and

(vi) $a, c$ and $b$ are collinear.

The theorem is a consequence of (6).

(8) ExT1:

Let us consider a non empty Tarski plane $S$ satisfying seven Tarski's geometry axioms, and points $a, b, c, d$ of $S$. Suppose $a \neq b$ and $a, b$ and $c$ are collinear and $a, b$ and $d$ are collinear. Then $a, c$ and $d$ are collinear. The theorem is a consequence of (4) and (5).

(9) Let us consider a non empty Tarski plane $S$ satisfying seven Tarski's geometry axioms, and points $a, b$ of $S$. Suppose Middle $(a, a, b)$ or Middle $(a, b, b)$ or Middle $(a, b, a)$. Then $a=b$.

(10) Suppose (Middle $(a, b, c)$ or Middle $(c, b, a))$ and $(a \neq b$ or $b \neq c)$. Then

(i) Line $(b, a)=\operatorname{Line}(b, c)$, and

(ii) $\operatorname{Line}(a, b)=\operatorname{Line}(b, c)$, and

(iii) Line $(a, b)=\operatorname{Line}(c, b)$, and

(iv) Line $(b, a)=\operatorname{Line}(c, b)$.

The theorem is a consequence of (9).

(11) Suppose $a \neq b$ and $c \neq c^{\prime}$ and $(c \in \operatorname{Line}(a, b)$ or $c \in \operatorname{Line}(b, a))$ and $\left(c^{\prime} \in \operatorname{Line}(a, b)\right.$ or $\left.c^{\prime} \in \operatorname{Line}(b, a)\right)$. Then

(i) Line $\left(c, c^{\prime}\right)=\operatorname{Line}(a, b)$, and

(ii) $\operatorname{Line}\left(c, c^{\prime}\right)=\operatorname{Line}(b, a)$, and

(iii) Line $\left(c^{\prime}, c\right)=\operatorname{Line}(b, a)$, and

(iv) $\operatorname{Line}\left(c^{\prime}, c\right)=\operatorname{Line}(a, b)$.

(12) $\operatorname{Middle}\left(\mathrm{S}_{p}(c), \mathrm{S}_{p}(b), \mathrm{S}_{p}\left(\left(\mathrm{~S}_{b}(c)\right)\right)\right)$.

\section{Right Angle}

Let $S$ be Tarski plane satisfying the axiom of congruence identity, the axiom of congruence symmetry, the axiom of congruence equivalence relation, the axiom of segment construction, the axiom of betweenness identity, and the axiom of SAS and $a, b, c$ be points of $S$. We say that $\mathrm{b}(a, b, c)$ if and only if

(Def. 1) $\overline{a c} \cong \overline{a \mathrm{~S}_{b}(c)}$.

From now on $S$ denotes Tarski plane satisfying seven Tarski's geometry axioms and $a, a^{\prime}, b, b^{\prime}, c, c^{\prime}$ denote points of $S$.

Now we state the propositions: 
(13) 8.2 SATZ:

If $\measuredangle(a, b, c)$, then $\measuredangle(c, b, a)$.

(14) $\mathrm{S}_{a}(a)=a$.

(15) 8.3 SATZ:

If $\measuredangle(a, b, c)$ and $a \neq b$ and $b, a$ and $a^{\prime}$ are collinear, then $\measuredangle\left(a^{\prime}, b, c\right)$. The theorem is a consequence of (14).

(16) 8.4 SATZ:

If $\measuredangle(a, b, c)$, then $\measuredangle\left(a, b, \mathrm{~S}_{b}(c)\right)$.

(17) 8.5 SATZ:

$\llcorner(a, b, b)$. The theorem is a consequence of (14).

(18) 8.6 SATZ:

If $b(a, b, c)$ and $b\left(a^{\prime}, b, c\right)$ and $c$ lies between $a$ and $a^{\prime}$, then $b=c$.

(19) 8.7 SATZ:

If $\llcorner(a, b, c)$ and $b(a, c, b)$, then $b=c$. The theorem is a consequence of (13), (17), (1), (7), (15), and (18).

(20) 8.8 SATZ:

If $\measuredangle(a, b, a)$, then $a=b$. The theorem is a consequence of $(13),(17)$, and (19).

(21) 8.9 SATZ:

If $\measuredangle(a, b, c)$ and $a, b$ and $c$ are collinear, then $a=b$ or $c=b$. The theorem is a consequence of (15) and (20).

(22) 8.10 SATZ:

If $\measuredangle(a, b, c)$ and $\triangle a b c \cong \triangle a^{\prime} b^{\prime} c^{\prime}$, then $\downarrow\left(a^{\prime}, b^{\prime}, c^{\prime}\right)$. The theorem is a consequence of (17), (1), and (3).

\section{Orthogonality}

Let $S$ be a non empty Tarski plane satisfying seven Tarski's geometry axioms, $A, A^{\prime}$ be subsets of $S$, and $x$ be a point of $S$. We say that $A \perp_{x} A^{\prime}$ if and only if (Def. 2) $A$ is a line and $A^{\prime}$ is a line and $x \in A$ and $x \in A^{\prime}$ and for every points $u$, $v$ of $S$ such that $u \in A$ and $v \in A^{\prime}$ holds $b(u, x, v)$.

We say that $A \perp A^{\prime}$ if and only if

(Def. 3) there exists a point $x$ of $S$ such that $A \perp_{x} A^{\prime}$.

Let $A$ be a subset of $S$ and $x, c, d$ be points of $S$. We say that $\overline{A, x} \perp \overline{c, d}$ if and only if

(Def. 4) $\quad c \neq d$ and $A \perp_{x} \operatorname{Line}(c, d)$.

Let $a, b, x, c, d$ be points of $S$. We say that $\overline{a, b} \perp_{x} \overline{c, d}$ if and only if 
(Def. 5) $\quad a \neq b$ and $c \neq d$ and Line $(a, b) \perp_{x} \operatorname{Line}(c, d)$.

Let $a, b, c, d$ be points of $S$. We say that $\overline{a, b} \perp \overline{c, d}$ if and only if (Def. 6) $\quad a \neq b$ and $c \neq d$ and Line $(a, b) \perp \operatorname{Line}(c, d)$.

From now on $S$ denotes a non empty Tarski plane satisfying seven Tarski's geometry axioms, $A, A^{\prime}$ denote subsets of $S$, and $x, y, z, a, b, c, c^{\prime}, d, u, p, q, q^{\prime}$ denote points of $S$.

Now we state the propositions:

(23) 8.12 SATZ:

$A \perp_{x} A^{\prime}$ if and only if $A^{\prime} \perp_{x} A$.

(24) 8.13 SATZ:

$A \perp_{x} A^{\prime}$ if and only if $A$ is a line and $A^{\prime}$ is a line and $x \in A$ and $x \in A^{\prime}$ and there exist points $u, v$ of $S$ such that $u \in A$ and $v \in A^{\prime}$ and $u \neq x$ and $v \neq x$ and $\downarrow(u, x, v)$. The theorem is a consequence of (15) and (13).

(25) 8.14 (I) SATZ:

If $A \perp A^{\prime}$, then $A \neq A^{\prime}$. The theorem is a consequence of (24) and (21).

\section{INTERSECTION OF LINES}

Let $S$ be a non empty Tarski plane, $A, B$ be subsets of $S$, and $x$ be a point of $S$. We say that $A, B$ intersect at $x$ if and only if

(Def. 7) $A$ is a line and $B$ is a line and $A \neq B$ and $x \in A$ and $x \in B$.

Now we state the propositions:

(26) 8.14 (II) SATZ:

$A \perp_{x} A^{\prime}$ if and only if $A \perp A^{\prime}$ and $A, A^{\prime}$ intersect at $x$. The theorem is a consequence of (25).

(27) 8.14 (III) SATZ:

If $A \perp_{x} A^{\prime}$ and $A \perp_{y} A^{\prime}$, then $x=y$. The theorem is a consequence of (25) and (26).

(28) If $a, b$ and $x$ are collinear and $\overline{a, b} \perp \overline{c, x}$, then $\overline{a, b} \perp_{x} \overline{c, x}$. The theorem is a consequence of (25) and (26).

(29) 8.15 SATZ:

If $a \neq b$ and $a, b$ and $x$ are collinear, then $\overline{a, b} \perp \overline{c, x}$ iff $\overline{a, b} \perp_{x} \overline{c, x}$. The theorem is a consequence of (28).

(30) 8.16 SATz:

Suppose $a \neq b$ and $a, b$ and $x$ are collinear and $a, b$ and $u$ are collinear and $u \neq x$. Then $\overline{a, b} \perp \overline{c, x}$ if and only if $a, b$ and $c$ are not collinear and $\measuredangle(c, x, u)$. The theorem is a consequence of $(29),(13),(21)$, and (24). 


\section{Perpendicular Foot}

Let $S$ be a non empty Tarski plane satisfying seven Tarski's geometry axioms and $a, b, c, x$ be points of $S$. We say that $x$ is perpendicular foot of $a, b, c$ if and only if

(Def. 8) $\quad a, b$ and $x$ are collinear and $\overline{a, b} \perp \overline{c, x}$.

Now we state the propositions:

(31) 8.18 SATZ - Uniqueness:

If $x$ is perpendicular foot of $a, b, c$ and $y$ is perpendicular foot of $a, b, c$, then $x=y$. The theorem is a consequence of (29), (13), and (19).

(32) Suppose $a, b$ and $c$ are not collinear and $a$ lies between $b$ and $y$ and $a \neq y$ and $y$ lies between $a$ and $z$ and $\overline{y z} \cong \overline{y p}$ and $y \neq p$ and $q^{\prime}=\mathrm{S}_{z}(q)$ and Middle $\left(c, x, c^{\prime}\right)$ and $c \neq y$ and $y$ lies between $q^{\prime}$ and $c^{\prime}$ and Middle $(y, p, c)$ and $y$ lies between $p$ and $q$ and $q \neq q^{\prime}$. Then $x \neq y$. The theorem is a consequence of (10) and (11).

In the sequel $S$ denotes a non empty Tarski plane satisfying Lower Dimension Axiom and seven Tarski's geometry axioms and $a, b, c, p, q, x, y, z, t$ denote points of $S$.

Now we state the propositions:

(33) 8.18 SATZ - Existence:

If $a, b$ and $c$ are not collinear, then there exists $x$ such that $x$ is perpendicular foot of $a, b, c$.

Proof: Consider $y$ such that $a$ lies between $b$ and $y$ and $\overline{a y} \cong \overline{a c}$. Consider $p$ such that Middle $(y, p, c)$. Consider $z$ such that $y$ lies between $a$ and $z$ and $\overline{y z} \cong \overline{y p}$. Consider $q$ such that $y$ lies between $p$ and $q$ and $\overline{y q} \cong \overline{y a}$. Set $q^{\prime}=\mathrm{S}_{z}(q)$. Consider $c^{\prime}$ such that $y$ lies between $q^{\prime}$ and $c^{\prime}$ and $\overline{y c^{\prime}} \cong \overline{y c}$. $a \neq y$. $\mathrm{b}(q, z, y)$. $\mathrm{b}(y, z, q)$. Consider $x$ such that Middle $\left(c, x, c^{\prime}\right) . y \neq p$. $c \neq y . q \neq q^{\prime} . c \neq x$.

(34) 8.20 LEMMA:

If $\measuredangle(a, b, c)$ and Middle $\left(\mathrm{S}_{a}(c), p, \mathrm{~S}_{b}(c)\right)$, then $\measuredangle(b, a, p)$ and if $b \neq c$, then $a \neq p$.

Proof: Set $d=\mathrm{S}_{b}(c)$. Set $b^{\prime}=\mathrm{S}_{a}(b)$. Set $c^{\prime}=\mathrm{S}_{a}(c)$. Set $d^{\prime}=\mathrm{S}_{a}(d)$. Set $p^{\prime}=\mathrm{S}_{a}(p)$. $\downarrow\left(b^{\prime}, b, c\right) \cdot \overline{b^{\prime} b} \cong \overline{b b^{\prime}} \cdot \overline{b^{\prime} c} \cong \overline{b c^{\prime}} \cdot \triangle b^{\prime} b c \cong \triangle b b^{\prime} c^{\prime} . \downarrow\left(b, b^{\prime}, c^{\prime}\right)$. $\mathrm{S}_{b^{\prime}}\left(c^{\prime}\right)=d^{\prime}$. IFS $\left(\begin{array}{c}c^{\prime}, p, d, b \\ d^{\prime}, p^{\prime}, c, b\end{array}\right)$. If $b \neq c$, then $a \neq p$.

(35) Suppose $a, b$ and $c$ are not collinear. Then there exists $p$ and there exists $t$ such that $\overline{a, b} \perp \overline{p, a}$ and $a, b$ and $t$ are collinear and $t$ lies between $c$ and $p$. The theorem is a consequence of (33), (29), (34), and (24).

(36) 8.21 SATZ:

If $a \neq b$, then there exists $p$ and there exists $t$ such that $\overline{a, b} \perp \overline{p, a}$ and $a, b$ 
and $t$ are collinear and $t$ lies between $c$ and $p$. The theorem is a consequence of $(35)$.

(37) If $a \neq b$ and $a \neq p$ and $\llcorner(b, a, p)$ and $\measuredangle(a, b, q)$, then $p, a$ and $q$ are not collinear. The theorem is a consequence of (13), (15), and (19).

(38) Let us consider a non empty Tarski plane $S$ satisfying Lower Dimension Axiom and seven Tarski's geometry axioms, and points $a, b, p, q, t$ of $S$. Suppose $a, p \leqslant b, q$ and $\overline{a, b} \perp \overline{q, b}$ and $\overline{a, b} \perp \overline{p, a}$ and $a, b$ and $t$ are collinear and $t$ lies between $q$ and $p$. Then there exists a point $x$ of $S$ such that $\operatorname{Middle}(a, x, b)$.

Proof: Consider $r$ being a point of $S$ such that $r$ lies between $b$ and $q$ and $\overline{a p} \cong \overline{b r}$. Consider $x$ being a point of $S$ such that $x$ lies between $t$ and $b$ and $x$ lies between $r$ and $p . a, b$ and $x$ are collinear. Consider $x^{\prime}$ being a point of $S$ such that Line $(a, b) \perp_{x^{\prime}} \operatorname{Line}(q, b)$. Consider $y$ being a point of $S$ such that Line $(a, b) \perp_{y} \operatorname{Line}(p, a) .\llcorner(q, b, a)$ and $q \neq b$ and $b, q$ and $r$ are collinear. $b(r, b, a) . b, a$ and $p$ are not collinear and $a, b$ and $q$ are not collinear.

(39) 8.22 SATz:

Let us consider a non empty Tarski plane $S$ satisfying Lower Dimension Axiom and seven Tarski's geometry axioms, and points $a, b$ of $S$. Then there exists a point $x$ of $S$ such that $\operatorname{Middle}(a, x, b)$. The theorem is a consequence of (36) and (38).

(40) 8.24 LEMMA:

Let us consider a non empty Tarski plane $S$ satisfying Lower Dimension Axiom and seven Tarski's geometry axioms, and points $a, b, p, q, r, t$ of $S$. Suppose $\overline{p, a} \perp \overline{a, b}$ and $\overline{q, b} \perp \overline{a, b}$ and $a, b$ and $t$ are collinear and $t$ lies between $p$ and $q$ and $r$ lies between $b$ and $q$ and $\overline{a p} \cong \overline{b r}$. Then there exists a point $x$ of $S$ such that

(i) Middle $(a, x, b)$, and

(ii) $\operatorname{Middle}(p, x, r)$.

Proof: Consider $x$ being a point of $S$ such that $x$ lies between $t$ and $b$ and $x$ lies between $r$ and $p . a, b$ and $x$ are collinear. Consider $x^{\prime}$ being a point of $S$ such that Line $(a, b) \perp_{x^{\prime}} \operatorname{Line}(q, b)$. Consider $y$ being a point of $S$ such that Line $(a, b) \perp_{y} \operatorname{Line}(p, a) .\llcorner(q, b, a)$ and $q \neq b$ and $b, q$ and $r$ are collinear. $b(r, b, a) . b, a$ and $p$ are not collinear and $a, b$ and $q$ are not collinear. 


\section{Additional Lemmas Needed by Otter: Chapter 8A}

Now we state the propositions:

(41) ExтCoL2:

Let us consider points $a, b, c, d, x, p, q$ of $S$. Suppose $c, d \in \operatorname{Line}(a, b)$ and $a \neq b$ and $c \neq d$. Then Line $(a, b)=\operatorname{Line}(c, d)$.

(42) ExtPerP:

Let us consider points $a, b, c, d, x, p, q$ of $S$. Suppose $c, d \in \operatorname{Line}(a, b)$ and $c \neq d$ and $\overline{a, b} \perp_{x} \overline{p, q}$. Then $\overline{c, d} \perp_{x} \overline{p, q}$. The theorem is a consequence of (11).

(43) EXTPERP2:

Let us consider points $a, b, c, d, p, q$ of $S$. Suppose $p, q \in \operatorname{Line}(a, b)$ and $a \neq b$ and $\overline{p, q} \perp \overline{c, d}$. Then $\overline{a, b} \perp \overline{c, d}$. The theorem is a consequence of (11).

(44) EXTPERP3:

Let us consider points $a, b, c, d$ of $S$. Suppose $a \neq b$ and $b \neq c$ and $c \neq d$ and $a \neq c$ and $a \neq d$ and $b \neq d$ and $\overline{b, a} \perp \overline{a, c}$ and $a, c$ and $d$ are collinear. Then $\overline{b, a} \perp \overline{a, d}$. The theorem is a consequence of (11).

(45) EXTPERP4:

Let us consider points $a, b, p, q$ of $S$. If $\overline{a, b} \perp \overline{p, q}$, then $\overline{a, b} \perp \overline{q, p}$.

(46) EXtPerp5:

Let us consider points $a, b, c, d, p, q$ of $S$. Suppose $p, q \in \operatorname{Line}(a, b)$ and $p \neq q$ and $\overline{a, b} \perp \overline{c, d}$. Then $\overline{p, q} \perp \overline{c, d}$. The theorem is a consequence of (11).

(47) ExtPerp5A:

Let us consider points $a, b, c, d, p, q$ of $S$. Suppose $a, b$ and $p$ are collinear and $a, b$ and $q$ are collinear and $p \neq q$ and $\overline{a, b} \perp \overline{c, d}$. Then $\overline{p, q} \perp \overline{c, d}$. The theorem is a consequence of (46).

(48) ExtPerp6:

Let us consider points $a, b, c, d, p, q$ of $S$. Suppose $p, q \in \operatorname{Line}(a, b)$ and $p \neq q$ and $a \neq b$ and $\overline{c, d} \perp \overline{p, q}$. Then $\overline{c, d} \perp \overline{a, b}$. The theorem is a consequence of (11).

\section{REFERENCES}

[1] Grzegorz Bancerek, Czesław Byliński, Adam Grabowski, Artur Korniłowicz, Roman Matuszewski, Adam Naumowicz, Karol Pąk, and Josef Urban. Mizar: State-of-the-art and beyond In Manfred Kerber, Jacques Carette, Cezary Kaliszyk, Florian Rabe, and Volker Sorge, editors, Intelligent Computer Mathematics, volume 9150 of Lecture Notes in Computer Science, pages 261-279. Springer International Publishing, 2015. ISBN 978-3319-20614-1. doi $10.1007 / 978-3-319-20615-8 \_17$. 
[2] Grzegorz Bancerek, Czesław Byliński, Adam Grabowski, Artur Korniłowicz, Roman Matuszewski, Adam Naumowicz, and Karol Pąk. The role of the Mizar Mathematical Library for interactive proof development in Mizar Journal of Automated Reasoning, 61(1):9-32, 2018. do1 $10.1007 / \mathrm{s} 10817-017-9440-6$

[3] Michael Beeson and Larry Wos. OTTER proofs in Tarskian geometry. In International Joint Conference on Automated Reasoning, volume 8562 of Lecture Notes in Computer Science, pages 495-510. Springer, 2014. doi 10.1007/978-3-319-08587-6_38

[4] Michael Beeson, Julien Narboux, and Freek Wiedijk. Proof-checking Euclid. Annals of Mathematics and Artificial Intelligence, Jan 2019. doi $10.1007 / \mathrm{s} 10472-018-9606-\mathrm{x}$

[5] Pierre Boutry, Gabriel Braun, and Julien Narboux. Formalization of the Arithmetization of Euclidean Plane Geometry and Applications. Journal of Symbolıc Computation, 90: 149-168, 2019. doi 10.1016/j.jsc.2018.04.007.

[6] Pierre Boutry, Charly Gries, Julien Narboux, and Pascal Schreck. Parallel postulates and continuity axioms: a mechanized study in intuitionistic logic using Coq. Journal of Automated Reasoning, 62(1):1-68, 2019.

[7] Roland Coghetto and Adam Grabowski. Tarski geometry axioms. Part III. Formalized Mathematics, 25(4):289-313, 2017. doi 10.1515/forma-2017-0028

[8] Sana Stojanovic Durdevic, Julien Narboux, and Predrag Janičić. Automated generation of machine verifiable and readable proofs: a case study of Tarski's geometry. Annals of Mathematics and Artificial Intelligence, 74(3-4):249-269, 2015.

[9] Adam Grabowski. Tarski's geometry modelled in Mizar computerized proof assistant. In Maria Ganzha, Leszek Maciaszek, and Marcin Paprzycki, editors, Proceedings of the 2016 Federated Conference on Computer Science and Information Systems (FedCSIS), volume 8 of ACSIS - Annals of Computer Science and Information Systems, pages 373-381, 2016. doi: $10.15439 / 2016 \mathrm{~F} 290$

[10] Adam Grabowski and Roland Coghetto. Tarski's geometry and the Euclidean plane in Mizar. In Joint Proceedings of the FM4M, MathUI, and ThEdu Workshops, Doctoral Program, and Work in Progress at the Conference on Intelligent Computer Mathematics 2016 co-located with the 9th Conference on Intelligent Computer Mathematics (CICM 2016), Biatystok, Poland, July 25-29, 2016, volume 1785 of CEUR-WS, pages 4-9, 2016.

[11] Haragauri Narayan Gupta. Contributions to the Axiomatic Foundations of Geometry. PhD thesis, University of California-Berkeley, 1965.

[12] Julien Narboux. Mechanical theorem proving in Tarski's geometry. In Francisco Botana and Tomas Recio, editors, Automated Deduction in Geometry, pages 139-156, Berlin, Heidelberg, 2007. Springer Berlin Heidelberg. ISBN 978-3-540-77356-6.

[13] William Richter, Adam Grabowski, and Jesse Alama. Tarski geometry axioms. Formalized Mathematics, 22(2):167-176, 2014. doi 10.2478/forma-2014-0017.

[14] Wolfram Schwabhäuser, Wanda Szmielew, and Alfred Tarski. Metamathematische Methoden in der Geometrie. Springer-Verlag, Berlin, Heidelberg, New York, Tokyo, 1983. 